\title{
Efficacy and Safety of Peroral Endoscopic Myotomy With Short Myotomy for Type I and II Achalasia
}

\author{
Yang Won Min \\ Department of Medicine, Samsung Medical Center, Sungkyunkwan University School of Medicine, Seoul, Korea
}

\author{
Article: Comparison of short versus long esophageal myotomy in cases with idiopathic achalasia: a randomized controlled \\ trial \\ Nabi Z, Ramchandani M, Sayyed M, et al \\ (J Neurogastroenterol Motil 2021;27:63-70)
}

Achalasia is a primary esophageal motor disorder in which there is degeneration of neurons in the wall of the esophagus, leading to the absence of peristalsis and impaired relaxation of the lower esophageal sphincter. ${ }^{1}$ The main clinical presentation is dysphagia for solids and liquids. The aim of treatment options is to reduce elevated lower esophageal sphincter pressure. Pneumatic dilation and laparoscopic Heller's myotomy have been widely performed with similar efficacy. $^{2}$

With the concept of natural orifice transluminal endoscopic surgery, endoscopic myotomy was reported by Pasricha et $\mathrm{al}^{3}$ in a porcine model in 2007. One year later, Inoue at Showa University Northern Yokohama Hospital conducted a preliminary clinical application of the modified Pascricha technique as peroral endoscopic myotomy (POEM) for esophageal achalasia. Given the excellent clinical outcomes, POEM has been performed as the primary and rescue treatment for achalasia worldwide, and the number of procedures is increasing. ${ }^{4,5}$

POEM consists of 4 consecutive steps: (1) mucosal incision for entry into the submucosa, (2) submucosal tunneling, (3) myotomy, and (4) closure of the mucosal entry. Among these steps, myotomy is the core procedure for POEM. There are debatable concerns on the myotomy procedure, including the orientation, depth, and length in performing a myotomy. The anterior or posterior esophageal wall could be selected as the entry point and direction of the myotomy. Selective circular myotomy or full-thickness myotomy could also be performed during the procedure. However, myotomy length is closely associated with treatment efficacy and is the most important factor.

In the first case series of POEM, authors experienced poor outcomes from early patients with a relatively short myotomy. ${ }^{6}$ From the following papers, the average myotomy length has been reported with an average of $10 \mathrm{~cm}$ to $14 \mathrm{~cm}$, including a $2-\mathrm{cm}$ or 3 -cm gastric myotomy, and type III achalasia needs a longer myotomy. Although the significance of adequate gastric myotomy is well known, the optimal length of esophageal myotomy has not been established. Given the short length of the high-pressure zone in type I and II achalasia, a short esophageal myotomy, including adequate gastric myotomy, would be sufficient for relieving dysphagia in patients with type I and II achalasia. In a previous study, ${ }^{7} 46$ achalasia patients who received short myotomy (mean, $5.4 \mathrm{~cm}$ ) showed excellent short-term (3 months) efficacy.

Nabi et $\mathrm{al}^{8}$ have published in this edition of the Journal of

Received: November 25, 2020 Revised: None Accepted: December 17, 2020

(.) This is an Open Access article distributed under the terms of the Creative Commons Attribution Non-Commercial License (http://creativecommons. org/licenses/by-nc/4.0) which permits unrestricted non-commercial use, distribution, and reproduction in any medium, provided the original work is properly cited.

*Correspondence: Yang Won Min, MD, PhD

Department of Medicine, Samsung Medical Center, Sungkyunkwan University School of Medicine, 81 Irwon-ro, Gangnam-gu, Seoul 06351, Korea

Tel: +82-2-3410-3409; Fax: +82-2-3410-6983; E-mail: yangwonee@gmail.com 
Neurogastroenterology and Motility their study that compared the outcomes of short ( $3 \mathrm{~cm}, \mathrm{n}=34$ ) versus long ( $6 \mathrm{~cm}$ and above, $\mathrm{n}$ $=37$ ) esophageal myotomy in patients undergoing POEM for type I and II achalasia $(\mathrm{n}=71)$ using a prospective randomized design. The mean length of esophageal myotomy differs between the 2 groups ( $2.76 \pm 0.41$ vs $7.97 \pm 2.40, P<0.001)$, while that of gastric myotomy did not differ $(2.70 \pm 0.73$ vs $2.84 \pm 0.63)$. Clinical success was comparable in both groups at 1 year. The mean operative time was shorter in the short myotomy group than in the long myotomy group $(44.03 \pm 13.78$ and $72.43 \pm 27.28$ minutes, respectively; $P<0.001)$. However, the incidence of adverse events did not differ between both groups. These results suggest that a long esophageal myotomy has no significant clinical impact on the outcomes of type I and II achalasia patients. Thus, physicians could perform a short myotomy in these patients, which increases the time efficiency. However, the present study is a non-inferiority trial with small samples. We need to interpret the results in the background of these limitations. For a concrete conclusion, further large-scale studies with long-term follow-up periods are warranted.

\section{Financial support: None.}

\section{Conflicts of interest: None.}

\section{References}

1. Spechler SJ, Castell DO. Classification of oesophageal motility abnormalities. Gut 2001;49:145-151.

2. Boeckxstaens GE, Annese V, des Varannes SB, et al. Pneumatic dilation versus laparoscopic Heller's myotomy for idiopathic achalasia. N Engl J Med 2011;364:1807-1816.

3. Pasricha PJ, Hawari R, Ahmed I, et al. Submucosal endoscopic esophageal myotomy: a novel experimental approach for the treatment of achalasia. Endoscopy 2007;39:761-764.

4. Jung HK, Hong SJ, Lee OY, et al. 2019 Seoul Consensus on Esophageal Achalasia Guidelines. J Neurogastroenterol Motil 2020;26:180-203.

5. Brewer Gutierrez OI, Moran RA, Familiari P, et al. Long-term outcomes of per-oral endoscopic myotomy in achalasia patients with a minimum follow-up of 4 years: a multicenter study. Endosc Int Open 2020;8:E650-E655.

6. Inoue H, Minami H, Kobayashi Y, et al. Peroral endoscopic myotomy (POEM) for esophageal achalasia. Endoscopy 2010;42:265-271.

7. Wang J, Tan N, Xiao Y, et al. Safety and efficacy of the modified peroral endoscopic myotomy with shorter myotomy for achalasia patients: a prospective study. Dis Esophagus 2015;28:720-727.

8. Nabi Z, Ramchandani M, Sayyed M, et al. Comparison of short versus long esophageal myotomy in cases with idiopathic achalasia: a randomized controlled trial. J Neurogastroenterol Motil 2021;27:63-70. 